\title{
Self-calibration Method and Pose Domain Determination of Light-Pen in a 3D Vision Coordinate Measurement System
}

\author{
Dongri Shan ${ }^{1,}$, Chenglong Zhang ${ }^{1}$, Peng Zhang ${ }^{2}$, Xiaofang Wang ${ }^{2}$, Dongmei He ${ }^{2}$, Yalu $\mathrm{Xu}^{1}{ }^{1}$, Maohui Zhou ${ }^{1}$ and \\ Guoqi Yu ${ }^{1}$ \\ 1 School of Mechanical Engineering, Qilu University of Technology (Shandong Academy of Sciences), Jinan, \\ 250300, China ; shandongri@qlu.edu.cn(D.S.); zcl17864188832@163.com (C.Z.) ; \\ tyut_qlut_xuyalu@126.com(Y.X.); 1043119049@stu.qlu.edu.cn(M.Z.);1043119037@stu.qlu.edu.cn(G.Y) \\ 2 School of Electrical Engineering and Automation, Qilu University of Technology (Shandong Academy of \\ Sciences), Jinan, 250300, China; zp@qlu.edu.cn(P.Z.); wxfmail2008@163.com(X.W); he.ferry@163.com(D.H.) \\ * Correspondence: shandongri@qlu.edu.cn; Tel.: +86-138-6406-5008
}

\begin{abstract}
Light pen 3D vision coordinate measurement systems are increasingly widely used due to their advantages, such as small size, convenient carrying and wide applicability. The posture of the light pen is an important factor affecting accuracy. The pose domain of the pen needs to be given so that the measurement system has a suitable measurement range to obtain more qualified parameters. The advantage of the self-calibration method is that the entire self-calibration process can be completed at the measurement site without any auxiliary equipment. After the system camera calibration is completed, we take several pictures of the same measurement point with different poses to obtain the conversion matrix of the picture, and then use spherical fitting, the generalized inverse method of least squares, and the principle of position invariance within the pose domain range. The combined stylus tip center self-calibration method calculates the actual position of the light pen probe. The experimental results show that the absolute error is stable below $0.0737 \mathrm{~mm}$ and that the relative error is stable below $0.0025 \mathrm{~mm}$. The experimental results verify the effectiveness of the method; the measurement accuracy of the system can meet the basic industrial measurement requirements.
\end{abstract}

Keywords: stylus tip center self-calibration; spherical fitting; pose domain; vision measurement system

\section{Introduction}

With the continuous development of human society, manufacturing has begun to flourish, and industrial measurement has become an extremely important link in the process of industrial development. With the development of industries that require high-precision real-time measurement in the automotive industry and aerospace industry, there are an increasing number of applications and requirements for workpiece measurement, and the requirements for workpiece measurement accuracy are increasing. At present, mainstream measurement technology is unable to keep up with ever-increasing measurement requirements. The traditional coordinate measuring machine (CMM)[1] cannot meet the online measurement requirements of some industrial sites due to its large size and limited measurement range. Indoor GPS, White light scanner[2, 3], optical laser trackers[46] and other common measurement equipment $[7,8]$ also have many shortcomings, such as low measurement efficiency, high cost, complex configuration, and poor portability. The shortcomings of modern measurement technology have limited the development of industry to a certain extent. Therefore, development of a new measurement technology has become a common expectation. Vision measurement systems[9] are mainly based on 
computer vision, including measurement technology, electronic technology, image processing technology, etc., creating a new measurement structure frame, reducing measurement limitations, increasing flexibility and convenience, and allowing workpiece measurement from multiple angles. Its small size has met with favorable response in modern manufacturing fields such as aerospace and automobiles. The light-pen-type 3D vision measurement system[10] based on machine vision is increasingly widely used because of its small size, convenient portability, flexible assembly according to on-site measurement requirements, and good applicability, and related products have already been put into production[11]. Of course, many scholars have their own unique insights into visual measurement systems and have achieved notable results[12-15].

The actual coordinates of the center of the light pen stylus in the coordinate system directly affect the measurement accuracy of the three-coordinate measuring system. In practical applications, the center of the light pen stylus is mostly taken as a theoretical value when the light pen is used for processing. Due to the processing error of the light pen, the center value directly affects the measurement accuracy. When measuring various measurement objects, such as some deep holes and grooves, it is necessary to replace the light pen probe with other angles or add an extension rod for measurement. The difference in force and the loss of multiple uses of the light pen also cause small changes in the coordinates, so the self-calibration of the stylus tip center is particularly important. Liu optimized an objective function based on the principle of position invariance and the generalized inverse method of the least squares solution of the nonlinear equations and obtained the position of the stylus tip center in the coordinate system[16, 17]. Zheng proposed a two-step calibration method for the center of a plane target probe[18]. According to the principle of position invariance, the objective optimization function is established, and the optimized correction value of the stylus tip center in the $X$ and $Y$ directions in the target coordinate system is solved by the Levenberg-Marquardt algorithm. Then, the error of the stylus tip center in the $\mathrm{Z}$ direction is solved. Zhang also adopted the principle of position invariance and, based on this, gave error constraint parameters[19].

The work of this paper is divided into the determination of the pose domain and the self-calibration method of the probe center based on spherical fitting. The posture of the light pen is an important factor affecting accuracy. The pose domain of the light pen needs to be given so that the measurement system has a suitable measurement range to obtain more qualified parameters. Therefore, a threshold measurement device is designed according to the measurement system used in the experiment. The main body is composed of a light pen, connecting rod, base, motor seat, coupling and other parts. The transmission device is composed of a Siemens servo motor and the corresponding drive. The experiment was completed on the built measurement system[20]. After the motor parameters and program settings are completed, the experiment is driven by the motor to drive the device of the mechanical structure to make precise angle changes. For each rotation of $1^{\circ}$, the corresponding experimental image is collected to obtain the measurement error at this angle, and the data are sorted to obtain the pose that meets the requirements area. In the experiment of the self-calibration method, methods such as the principle of position invariance[17] are used. After the camera calibration is completed[21-24], the light pen probe collects multiple sets of images in different poses that meet the threshold measurement requirements under the CCD camera and uses the conversion relationship of each image to fit the same feature point on a sphere with the center of the probe as the center of the sphere, as shown in Figure 2. In this way, the actual position of the center of the probe is obtained. 




Figure 1. The principle of position invariance



Figure 2. Schematic diagram of the self-calibration method

\section{Self-calibration method of the stylus tip center}

\subsection{Light-pen-type 3D vision measurement system}

The light-pen-type vision measurement system is mainly composed of three parts: a CCD industrial camera capable of imaging infrared rays, a computer and light pen, as shown in Figure 3. The light pen consists of 8 infrared LEDs and a detachable ruby ball probe. Figure 4 shows several types of probes. During the measurement, the light pen image is taken by the CCD industrial camera and transmitted to the computer for related calculations, and finally, the measurement result is obtained.

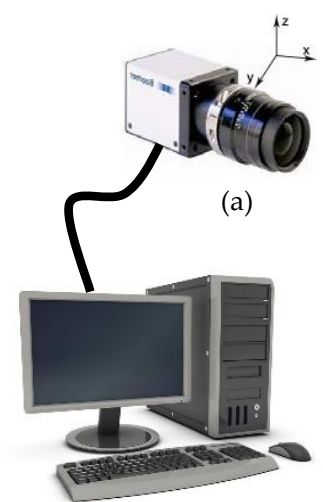

(b)

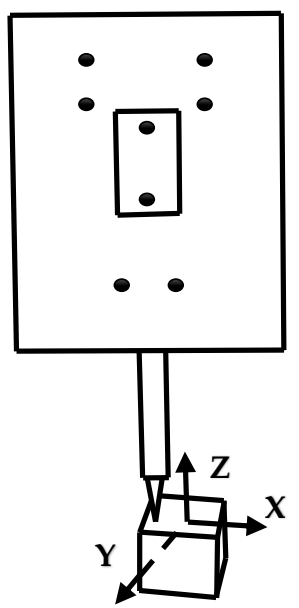

(c)

Figure 3. System composition diagram: (a)camera(b)computer(c)light pen 


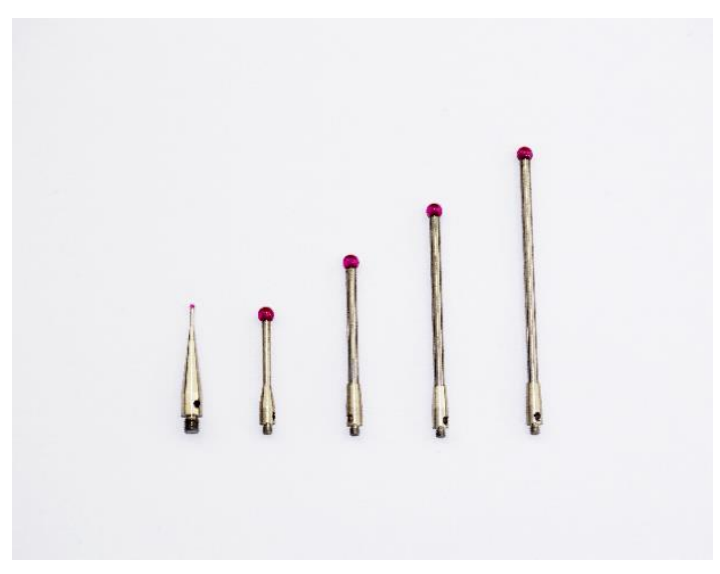

Figure 4. Light pen stylus

The measurement process of the measurement system is as follows: After the camera's internal parameters are calibrated[25], the user places the measured object and the light pen in the range that the CCD camera can shoot, the user presses the shooting button, the CCD camera takes a set of pictures, and the world coordinates of the measured object can be obtained. For the coordinates in the system, the distance between the two points can be measured by placing the light pen on another point in the same way.

\subsection{Establishment of the coordinate system}

The visual coordinate measurement system contains three coordinate systems:

1. Image coordinate system $\left(\mathrm{O}_{0}-\mathrm{XY}\right)$ :

The coordinate system is established on the imaging surface of the CCD camera, with the center of the imaging surface as the coordinate origin. The $\mathrm{x}$-axis and y-axis are respectively parallel to the horizontal and vertical imaging directions of the CCD camera, and parallel to the $\mathrm{O}_{1} \mathrm{UV}$ plane (unit: pixel).

2. Camera coordinate system ( $\left.\mathrm{O}_{1-\mathrm{uvw}}\right)$ :

The camera coordinate system is established on the industrial camera, and the origin of the camera coordinate system is the optical center of the industrial camera. The plane formed by the camera coordinate system is also parallel to the $\mathrm{O}_{1} \mathrm{UV}$ plane (unit: $\mathrm{mm}$ ).

3. Light pen coordinate system $\left(\mathrm{O}_{2}-\mathrm{xyz}\right)$ :

The theoretical value of the center of the light pen stylus is the coordinate origin, and the direction of the light pen shaft is the y-axis; the lower plane perpendicular to the six characteristic points of the light pen is the z-axis, and the $x$ axis is determined by the righthand rule (unit: $\mathrm{mm}$ ).

\subsection{Parameter solving}

Before measurement, the stylus tip center in the light pen coordinate system should be calibrated in advance. During the measurement, the images of the LED targets are captured by a CCD camera, and the computer processes the images to obtain the center coordinates of the center position of the target as a known quantity for calculating the rotation matrix R and the translation vector T[20]. The coordinates of the stylus tip center corresponding to the measured position in the camera coordinate system can be obtained by (1). Transformation from the camera coordinate system to the light pen coordinate system occurs as follows:

$$
\left[\begin{array}{c}
u \\
v \\
w
\end{array}\right]=\left[\begin{array}{llll}
r_{1} & r_{2} & r_{3} & t_{x} \\
r_{4} & r_{5} & r_{6} & t_{y} \\
r_{7} & r_{8} & r_{9} & t_{z}
\end{array}\right] \cdot\left[\begin{array}{c}
x \\
y \\
z \\
1
\end{array}\right]
$$


Here, $\left[\begin{array}{ccc}r_{1} & r_{2} & r_{3} \\ r_{4} & r_{5} & r_{6} \\ r_{7} & r_{8} & r_{9}\end{array}\right]$ is the rotation matrix $\mathrm{R}$ of the image, and $\left[\begin{array}{c}t_{x} \\ t_{y} \\ t_{z}\end{array}\right]$ is the displacement matrix $\mathrm{T}$ of the image.

We establish the relationship between the camera coordinate system and the pixel coordinate system:

$$
s \cdot\left[\begin{array}{c}
X \\
Y \\
1
\end{array}\right]=\left[\begin{array}{lll}
f & 0 & 0 \\
0 & f & 0 \\
0 & 0 & f
\end{array}\right]\left[\begin{array}{c}
u \\
v \\
w
\end{array}\right]
$$

The conversion between the camera coordinate system and the pixel coordinate system is carried out by Equation (2); $f$ is the focal length, $X=\frac{f}{w} u, Y=\frac{f}{w} v$;

We establish the linear equation set of the $\mathrm{j}$-th feature point in the image:

$$
\begin{gathered}
s_{j} \cdot\left[\begin{array}{c}
X_{j} \\
Y_{j} \\
1
\end{array}\right]=\mathrm{t}_{z}\left[\begin{array}{cccc}
a_{1} & a_{2} & a_{3} & a_{4} \\
a_{5} & a_{6} & a_{7} & a_{8} \\
a_{9} & a_{10} & a_{11} & 1
\end{array}\right]\left[\begin{array}{c}
x_{j} \\
y_{j} \\
z_{j} \\
1
\end{array}\right] \\
\operatorname{let} \mathrm{t}_{z}\left[\begin{array}{llll}
a_{1} & a_{2} & a_{3} & a_{4} \\
a_{5} & a_{6} & a_{7} & a_{8} \\
a_{9} & a_{10} & a_{11} & 1
\end{array}\right]=\left[\begin{array}{ccc}
f & 0 & 0 \\
0 & f & 0 \\
0 & 0 & f
\end{array}\right]\left[\begin{array}{c}
u \\
v \\
w
\end{array}\right]=\left[\begin{array}{ccc}
f & 0 & 0 \\
0 & f & 0 \\
0 & 0 & f
\end{array}\right]\left[\begin{array}{llll}
r_{1} & r_{2} & r_{3} & t_{x} \\
r_{4} & r_{5} & r_{6} & t_{y} \\
r_{7} & r_{8} & r_{9} & t_{z}
\end{array}\right] \\
s_{j}=r_{7} x_{j}+r_{8} y_{j}+r_{9} z_{j}+t_{z}=t_{z}\left(a_{9} x_{j}+a_{10} y_{j}+a_{11} z_{j}+1\right)=t_{z} \zeta_{j} \\
\text { Equation (3) is equivalent to: } \zeta_{j} \cdot\left[\begin{array}{c}
X_{c j} \\
Y_{c j} \\
1
\end{array}\right]=\left[\begin{array}{llll}
a_{1} & a_{2} & a_{3} & a_{4} \\
a_{5} & a_{6} & a_{7} & a_{8} \\
a_{9} & a_{10} & a_{11} & 1
\end{array}\right] \cdot\left[\begin{array}{c}
x_{j} \\
y_{j} \\
z_{j} \\
1
\end{array}\right]
\end{gathered}
$$

After solving the linear equations, the parameters are separated to obtain the initial values of $R$ and $T$.

In the ideal perspective imaging process, the solution obtained by the above formula is feasible because there is no error in the coordinate values of the object point and the image point in each coordinate system, and the calculated $\mathrm{R}$ satisfies the orthogonal system constraint. However, in the actual environment, due to various factors, such as the camera parameter calibration error, the image plane position extraction error of the control point center, and the coordinate value calibration error of the control point center in the coordinate system, the R matrix does not satisfy the orthogonal constraint relationship. Consequently, the matrix $\mathrm{T}$ also has a large error, so the initial values of $\mathrm{R}$ and $\mathrm{T}$ can be obtained by the linear equation solving method, and then the optimal solution of $\mathrm{R}$ and $\mathrm{T}$ can be obtained by the Newton-Gaussian iteration method[33].

\subsection{Self-calibration method of the stylus tip center}

After $\mathrm{R}$ and $\mathrm{T}$ are determined, because the distance between the characteristic point of the light pen and the actual position of the stylus tip center coordinate is a fixed value, according to the generalized inverse method of the nonlinear least square method, we can obtain: 


$$
\left(\mathrm{x}_{\mathrm{ij}}-\mathrm{x}_{0}\right)^{2}+\left(\mathrm{y}_{\mathrm{ij}}-\mathrm{y}_{0}\right)^{2}+\left(\mathrm{z}_{\mathrm{ij}}-\mathrm{z}_{0}\right)^{2}=\mathrm{d}_{i j}^{2}
$$

Here, $i$ represents the $i$-th image, and $j$ represents the $j$-th feature point

The coordinate positions of the pictures in the coordinate system are different, but the distance from the actual position of the center coordinate of the probe is the same, so the same feature point of the $\mathrm{n}$ groups of pictures in the same spatial coordinate system (based on the probe and the actual position of the center coordinate) is the center of the sphere, and the constant distance between the characteristic point and the stylus tip center is the radius of the sphere. The $8 \mathrm{n}$ coordinates of $\mathrm{n}$ sets of pictures of the fitted 8 spheres are used to calculate the actual position of the center coordinates of the stylus.

We obtain the residual formula:

$$
F=\sum_{\mathrm{i}=1}^{n}\left[\left(x_{i}-x_{0}\right)^{2}+\left(y_{i}-y_{0}\right)^{2}+\left(z_{i}-z_{0}\right)^{2}-d^{2}\right]^{2}
$$

The corresponding partial derivatives are

$$
\begin{aligned}
& \frac{\partial F}{\partial x}=-4 \sum_{i=1}^{n}\left(x_{i}-x_{0}\right)\left[\left(x_{i}-x_{0}\right)^{2}+\left(y_{i}-y_{0}\right)^{2}+\left(z_{i}-z_{0}\right)^{2}-d^{2}\right] \\
& \frac{\partial F}{\partial y}=-4 \sum_{i=1}^{n}\left(y_{i}-y_{0}\right)\left[\left(x_{i}-x_{0}\right)^{2}+\left(y_{i}-y_{0}\right)^{2}+\left(z_{i}-z_{0}\right)^{2}-d^{2}\right] \\
& \frac{\partial F}{\partial z}=-4 \sum_{i=1}^{n}\left(z_{i}-z_{0}\right)\left[\left(x_{i}-x_{0}\right)^{2}+\left(y_{i}-y_{0}\right)^{2}+\left(z_{i}-z_{0}\right)^{2}-d^{2}\right] \\
& \frac{\partial F}{\partial d}=-4 d \sum_{i=1}^{n}\left[\left(x_{i}-x_{0}\right)^{2}+\left(y_{i}-y_{0}\right)^{2}+\left(z_{i}-z_{0}\right)^{2}-d^{2}\right]
\end{aligned}
$$

We insert the coordinate values, set the partial derivative equal to zero, construct a system of equations, and solve it to obtain the actual position of the center coordinate of the probe.

\subsection{Self-calibration steps}

1. After the measurement system is built, use the spatial coordinates of the characteristic points of the light pen measured by CMM as the initial value.

2. Use the principle of position invariance to shoot images of different poses. In view of the particularity of the self-calibration method, the image pose should be changed as much as possible within the threshold range to make the result more accurate.

3. After obtaining at least 8 sets of pictures within the threshold range, calculate $R$ and $T$ corresponding to each image by Equations (1)-(5).

4. After R and T are determined, construct Equations (6)-(8), and solve them.

5. Obtain the actual position of the stylus tip center coordinate in the light pen coordinate system.

\section{Experiment}

\subsection{Pose domain experiment}

We performed the threshold measurement experiment according to the above method.

When rotating around the $\mathrm{X}$-axis, the angle between the light pen and the positive half axis of the $\mathrm{Y}$-axis (pitch) is $41^{\circ} \sim 159^{\circ}$, and there is a clear and recognizable image.

When rotating around the $\mathrm{Y}$-axis, the angle between the light pen and the positive half axis of the $\mathrm{X}$-axis (yaw) is $0^{\circ} \sim 180^{\circ}$, and there is a clear and recognizable image.

When rotating around the $\mathrm{Z}$ axis, the angle between the light pen and the positive half axis of the $\mathrm{X}$-axis (roll) is $0^{\circ} \sim 45^{\circ}$ and $136^{\circ} \sim 180^{\circ}$, and there is a clear and recognizable image. 
Due to the processing error of the mechanical device and the measurement error of the measuring equipment, we use $1^{\circ}$ as the unit of the data under the premise of ensuring that the error does not affect the experimental results. The motor drives the light pen to the measurement position, takes a stable image, calculates the measurement accuracy, and then rotates one degree to take the image. Every time it rotates by one degree, the camera collects 15 groups of images within the angle, analyzes and processes the data, and compares the obtained measurement results with the real values to obtain a difference. The graphs are shown in Figures 5-7.

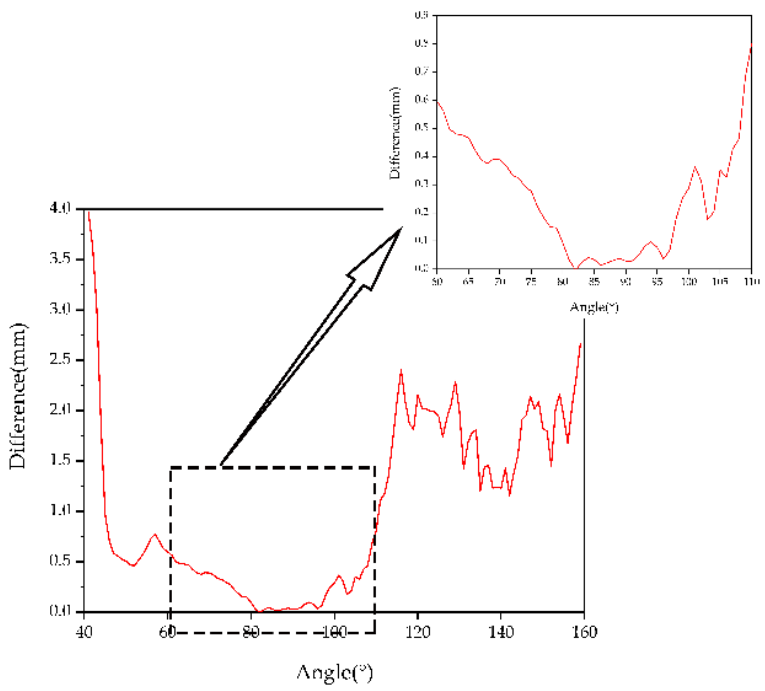

Figure 5. Pitch angle measurement results



Figure 6. Yaw angle measurement results
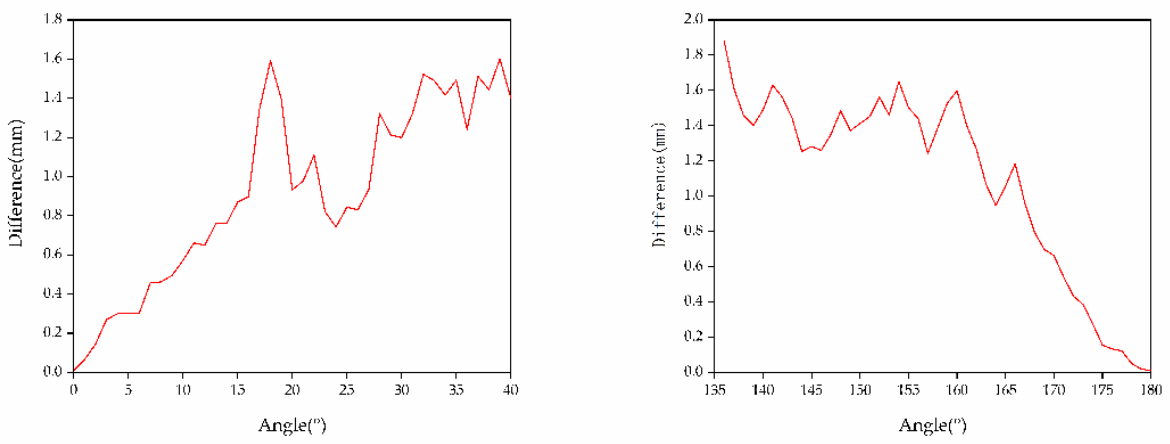
(a)

(b)

Figure 7. Tilt angle measurement results: (a)is the results of $0^{\circ} \sim 40^{\circ}(\mathrm{b})$ is the results of $136^{\circ} \sim 180^{\circ}$

When the pitch angle range is $80^{\circ} \sim 97^{\circ}$, the yaw angle is $80^{\circ} \sim 101^{\circ}$, and the tilt angle is $0^{\circ} \sim 2^{\circ}$ and $177^{\circ} \sim 180^{\circ}$, a certain measurement accuracy can be guaranteed to meet the measurement requirements. Satisfactory experimental results were obtained.

\subsection{Self-calibration method}

3.2.1. Self-calibration measurement experiment

We carried out the self-calibration experiment according to the method steps in this article. The self-calibration method was incorporated into the light pen measurement system for measurement comparison experiments, and each light pen performed 10 measurement experiments. Compare the measured value with the real value $(30.0317 \mathrm{~mm})$ to get the absolute error and relative error. The measurement results are shown in Table 1.

Table 1. Self-calibration experiment

\begin{tabular}{cccccc}
\hline Stylus type & A & B & C & D & E \\
\hline 1 & 29.989 & 30.331 & 30.272 & 30.242 & 29.923 \\
2 & 29.870 & 30.305 & 30.248 & 30.214 & 29.853 \\
3 & 30.083 & 30.294 & 30.254 & 30.244 & 30.058 \\
4 & 29.733 & 29.865 & 29.828 & 29.820 & 29.750 \\
5 & 29.905 & 29.792 & 29.770 & 29.794 & 29.939 \\
6 & 30.135 & 30.058 & 30.051 & 30.080 & 30.127 \\
7 & 29.994 & 29.773 & 29.764 & 29.801 & 30.018 \\
8 & 29.877 & 29.560 & 29.561 & 29.609 & 29.869 \\
9 & 30.187 & 29.892 & 29.900 & 29.956 & 30.209 \\
10 & 30.036 & 29.948 & 29.931 & 29.958 & 30.046 \\
\hline Average value & 29.981 & 29.982 & 29.958 & 29.972 & 29.979 \\
\hline Absolute error & 0.051 & 0.050 & 0.074 & 0.060 & 0.053 \\
\hline Relative error & $0.17 \%$ & $0.17 \%$ & $0.25 \%$ & $0.20 \%$ & $0.18 \%$ \\
\hline
\end{tabular}

The measured values of the 5 light pen styluses are displayed on lines 2 to 11 . The last three lines show the average, absolute error and relative error. The measurement results of each stylus in the ten images are represented by 1 to 10 lines. As shown in Table 1 , the absolute error of the probe of each light pen is stabilized below $0.0737 \mathrm{~mm}$, and the relative error is stabilized below $0.0025 \mathrm{~mm}$. The experimental results meet the basic industrial measurement requirements, verifying the effectiveness of this method.

3.2.2. Single-point repeatability experiment

We put the light pen stylus in a standard cone, and under the premise of keeping the position of the light pen stylus unchanged, we rotate the position of the light pen to perform a single-point repeatability experiment.

Table 2. Single-point repeatability experiment

\begin{tabular}{|c|c|c|c|c|c|c|c|c|c|c|c|c|c|}
\hline & Test & 1 & 2 & 3 & 4 & 5 & 6 & 7 & 8 & 9 & 10 & AVE & STD \\
\hline \multirow{3}{*}{ A } & $\mathrm{u}$ & 78.995 & 78.995 & 79.014 & 79.004 & 78.997 & 78.997 & 78.983 & 79.002 & 78.990 & 78.995 & 78.997 & 0.008 \\
\hline & $\mathrm{v}$ & 79.967 & 79.975 & 79.965 & 79.968 & 79.961 & 79.964 & 79.982 & 79.956 & 79.979 & 79.980 & 79.970 & 0.009 \\
\hline & $\mathrm{w}$ & 790.029 & 789.994 & 790.040 & 790.026 & 790.047 & 790.030 & 789.965 & 790.083 & 789.986 & 789.948 & 790.015 & 0.041 \\
\hline \multirow{2}{*}{ B } & $\mathrm{u}$ & 79.061 & 79.074 & 79.054 & 79.066 & 79.068 & 79.071 & 79.056 & 79.064 & 79.068 & 79.047 & 79.063 & 0.008 \\
\hline & $\mathrm{v}$ & 80.262 & 80.250 & 80.275 & 80.247 & 80.261 & 80.243 & 80.268 & 80.260 & 80.271 & 80.269 & 80.261 & 0.011 \\
\hline
\end{tabular}




\begin{tabular}{|c|c|c|c|c|c|c|c|c|c|c|c|c|c|}
\hline & $\mathrm{w}$ & 790.209 & 790.262 & 790.205 & 790.261 & 790.220 & 790.271 & 790.236 & 790.277 & 790.216 & 790.219 & 790.238 & 0.028 \\
\hline \multirow{3}{*}{ C } & $\mathrm{u}$ & 79.094 & 79.096 & 79.090 & 79.094 & 79.103 & 79.094 & 79.093 & 79.102 & 79.096 & 79.097 & 79.096 & 0.004 \\
\hline & $\mathrm{v}$ & 80.626 & 80.604 & 80.619 & 80.634 & 80.616 & 80.625 & 80.627 & 80.611 & 80.628 & 80.609 & 80.620 & 0.01 \\
\hline & $\mathrm{w}$ & 790.286 & 790.298 & 790.263 & 790.222 & 790.283 & 790.235 & 790.231 & 790.302 & 790.250 & 790.289 & 790.266 & 0.03 \\
\hline \multirow{3}{*}{$\mathrm{D}$} & $\mathrm{u}$ & 78.907 & 78.900 & 78.897 & 78.900 & 78.906 & 78.909 & 78.899 & 78.887 & 78.899 & 78.906 & 78.901 & 0.006 \\
\hline & $\mathrm{v}$ & 79.347 & 79.352 & 79.352 & 79.348 & 79.340 & 79.340 & 79.345 & 79.357 & 79.349 & 79.342 & 79.347 & 0.005 \\
\hline & $\mathrm{w}$ & 789.472 & 789.444 & 789.425 & 789.474 & 789.508 & 789.518 & 789.481 & 789.404 & 789.467 & 789.509 & 789.470 & 0.037 \\
\hline \multirow{3}{*}{ E } & $\mathrm{u}$ & 79.155 & 79.166 & 79.166 & 79.159 & 79.178 & 79.167 & 79.175 & 79.167 & 79.164 & 79.169 & 79.167 & 0.007 \\
\hline & $\mathrm{v}$ & 80.843 & 80.869 & 80.860 & 80.845 & 80.861 & 80.859 & 80.856 & 80.860 & 80.868 & 80.883 & 80.860 & 0.012 \\
\hline & $\mathrm{W}$ & 790.859 & 790.855 & 790.882 & 790.908 & 790.881 & 790.845 & 790.909 & 790.907 & 790.864 & 790.843 & 790.875 & 0.026 \\
\hline
\end{tabular}

The experimental results of 5 light pen styluses are shown in Table 2. The coordinates of the center point of each light pen stylus in the ten images are represented by columns 1 to 10. Single point repeatability is expressed by the standard deviation of ten center point coordinates. As shown in the previous column, the probe repeatability of each light pen does not exceed 0.041 , which verifies the effectiveness of the self-calibration method.

\section{Conclusions}

This paper proposes a new self-calibration method, determines the pose domain of the measurement system through experiments, and describes the self-calibration process of the measurement system in detail. In the pose domain, the actual position of the light pen probe is calculated by a combination of spherical fitting, the generalized inverse method of the least square method, and the principle of position invariance. The experimental results are satisfactory, and the measurement accuracy after calibration is notably better than that before calibration. In future research, we plan to explore other factors that improve the measurement accuracy of the light-pen-type 3D vision measurement system.

Author Contributions: Conceptualization, D.S. and C.Z.; methodology, C.Z and Y.X.; formal analysis,C.Z.; resources, D.S.; data curation, C.Z.; writing-original draft preparation, C.Z.; writingreview and editing, P.Z.,X.W.,D.H. ; Validation, G.Y. and M.Z.; Supervision, D.S.

Funding: This research was funded by the Project of Shandong Provincial Major Scientific and Technological Innovation, grant No.2019JZZY010444, No.2019TSLH0315, in part by the Project of 20 Policies of Facilitate Scientific Research in Jinan Colleges, grant No.2019GXRC063, in part by the Project of Shandong Province Higher Educational Science and Technology Program, grant No.J18KA345, and in part by the Natural Science Foundation of Shandong Province of China, grant No.ZR2020MF138.

Conflicts of Interest: The authors declare no conflict of interest.

\section{References}

1. Brezina, I., Coordinate measuring machines and systems: John A. Bosch (Ed.), Marcel Dekker, Inc., New York/Basel/Hong Kong, 1995, 1st Edition, xi + 444 pp., ISBN 0-8247-9581-4, Measurement, 1997. 20(4): p. 287.

2. Milroy, M.J., et al., Reverse engineering employing a 3D laser scanner: A case study. The International Journal of Advanced Manufacturing Technology, 1996. 12(2): p. 111-121.

3. Chen, J., et al., 3D shape modeling using a self-developed hand-held 3D laser scanner and an efficient HT-ICP point cloud registration algorithm. Optics \& Laser Technology, 2013. 45: p. 414-423.

4. Vincze, M., J.P. Prenninger, and H. Gander, A Laser Tracking System to Measure Position and Orientation of Robot End Effectors Under Motion. The International Journal of Robotics Research, 1994. 13(4): p. 305-314.

5. Mayer, J.R.R. and G.A. Parker, A portable instrument for 3-D dynamic robot measurements using triangulation and laser tracking. IEEE Transactions on Robotics and Automation, 1994. 10(4): p. 504-516. 
6. Xie, Z., Z. Zhang, and M. Jin, Development of a multi-view laser scanning sensor for reverse engineering. Measurement Science and Technology, 2006. 17(8): p. 2319-2327.

7. Santolaria, J., et al., Modelling and calibration technique of laser triangulation sensors for integration in robot arms and articulated arm coordinate measuring machines. Sensors (Basel), 2009. 9(9): p. 7374-96.

8. Zhou, N., et al., iGPS Measurement Network Multi-Station Arrangement Design. Applied Mechanics and Materials, 2013. 443: p. 223-227.

9. Estler, T., et al., Large-Scale Metrology - An Update. Cirp Annals-manufacturing Technology - CIRP ANN-MANUF TECHNOL, 2002. 51: p. 587-609.

10. Zhi-jiang, Z., et al. Probe imaging vision coordinate measuring system using single camera. in Proc.SPIE. 1998.

11. Fraser, C., Innovations in Automation for Vision Metrology Systems. The Photogrammetric Record, 2003. 15: p. $901-911$.

12. Wang, S., S. Liu, and Q. Mao, A CMM-Based Method of Control Point Position Calibration for Light Pen Coordinate Measuring System. Sensors (Basel), 2020. 20(19).

13. Zhang, R., S.G. Liu, and H.Y. Wang, Wireless Control for Pointolite in Light-Pen CMMs. Applied Mechanics and Materials, 2014. 602-605: p. 2217-2220.

14. Fu, S., et al., A flexible approach to light pen calibration for a monocular-vision-based coordinate measuring system. Measurement Science and Technology, 2014. 25(12).

15. Alblalaihid, K., et al., Performance Assessment of a New Variable Stiffness Probing System for Micro-CMMs. Sensors (Basel), 2016. 16(4).

16. Liu, S.G., et al., A Portable 3D Vision Coordinate Measurement System Using a Light Pen. Key Engineering Materials, 2005. 295296: p. 331-336.

17. Liu, S., et al., Portable Light Pen 3D Vision Coordinate Measuring System- Probe Tip Center Calibration. Measurement Science Review, 2013. 13: p. 194-199.

18. Zheng, X., M. Zhao, and S. Feng, Two-Step Calibration of Probe Tip Center of Planar Target. Laser\&Optoelectronics Progress, 2018.

19. Zhang, R., et al., Stylus Tip Center Position Self-Calibration Based on Invariable Distances in Light-Pen Systems. Sensors (Basel), 2017. 17(1).

20. Shan, D., et al., The Impact of Control Point Dispersion on Measurement Accuracy in a New Type of Light-Pen Coordinate Measuring System. IEEE Access, 2021. 9: p. 76015-76023.

21. Tsai, R., A versatile camera calibration technique for high-accuracy 3D machine vision metrology using off-the-shelf TV cameras and lenses. IEEE Journal on Robotics and Automation, 1987. 3(4): p. 323-344.

22. Heikkila, J., Geometric camera calibration using circular control points. IEEE Transactions on Pattern Analysis and Machine Intelligence, 2000. 22(10): p. 1066-1077.

23. Svoboda, T., D. Martinec, and T. Pajdla, A Convenient Multicamera Self-Calibration for Virtual Environments. Presence: Teleoperators and Virtual Environments, 2005. 14(4): p. 407-422.

24. Zhengyou, Z., Camera calibration with one-dimensional objects. IEEE Transactions on Pattern Analysis and Machine Intelligence, 2004. 26(7): p. 892-899.

25. Zhang, Z., A flexible new technique for camera calibration. IEEE Transactions on Pattern Analysis and Machine Intelligence, 2000. 22(11): p. 1330-1334. 